\title{
3-Hydroxy-3-methylglutaric aciduria with bilateral basal ganglia lesion: A case report
}

\author{
XIAOSHENG HAO ${ }^{1}$, JIANGTAO WANG ${ }^{1}$, SONGYAN LIU $^{2}$, YINBO CHEN $^{1}$, YAN ZHANG $^{1}$ and YUNPENG HAO $^{1}$ \\ ${ }^{1}$ Department of Pediatric Neurology, The First Hospital of Jilin University, Changchun, Jilin 130021; \\ ${ }^{2}$ Department of Neurology, The P.R. China-Japan Union Hospital, Changchun, Jilin 130033, P.R. China
}

Received February 12, 2015; Accepted March 18, 2016

DOI: $10.3892 /$ etm.2016.3243

\begin{abstract}
Hydroxy-3-methylglutaric aciduria (3-HMG, OMIN 246450) is a rare autosomal recessive metabolic disorder caused by a deficiency of 3-hydroxy-3-methylglutaryl-CoA lyase, a key enzyme in leucine metabolism and ketone body synthesis. Acute episodes of 3-HMG may be triggered by fasting or infection, and symptoms include vomiting, diarrhea, lethargy and hypotonia. If left untreated, prolonged hypoglycemia and metabolic acidosis may cause breathing problems, seizures, and coma. In addition, 3-HMG is associated with damage to the central nervous system, and there are several reports of white matter abnormality or cerebral atrophy. The presence of bilateral basal ganglia damage in 3-HMG has been rarely reported. Here, we present a case report of a 20-month old male with severe 3-HMG and prominent bilateral lesions in the basal ganglia.
\end{abstract}

\section{Introduction}

3-Hydroxy-3-methylglutaric aciduria (3-HMG), also called 3-hydroxy-3-methylglutaryl-CoA lyase (HMG-CoA lyase or HL) deficiency, is an autosomal recessive disorder of the metabolism (1). This disease was first described in 1976 in a 7-month old boy from Australia (2). His major symptoms included vomiting, cyanosis, apnea, metabolic acidosis, hypoglycemia, and an increase in urine 3-hydroxy-3-methylglutaric acid (2). To date, $\sim 100$ cases have been reported worldwide, with an incidence of $<1 / 100,000$ among live newborns (3). The highest prevalence of the disease is in Saudi Arabia (3), and it is rarely observed in other populations, with only two reports from Taiwan and two from mainland China $(4,5)$.

HL catalyzes the final step of the mitochondrial ketogenic pathway and leucine catabolism by cleaving HMG-CoA to yield acetyl-CoA and acetoacetic acid (6). In the absence of

Correspondence to: Dr Yunpeng Hao, Department of Pediatric Neurology, The First Hospital of Jilin University, 71 Xinmin Street, Changchun, Jilin 130021, P.R. China

E-mail: gensheng201@163.com

Keywords: bilateral basal ganglialesion,3-hydroxy-3-methylglutaric aciduria, 3-hydroxy-3-methylglutaryl-CoA lyase
$\mathrm{HL}$, leucine cleavage is incomplete, resulting in accumulation of metabolic acids and compromised ketone body synthesis (6). Elevated levels of metabolic acids often cause liver damage and/or hepatomegaly in 3-HMG patients (7). Normally, during periods of fasting or infection, generation of ketone bodies in mitochondria provide an alternative energy source for the brain, heart and kidney (6). Impairments in ketogenesis may lead to hyperammonemia, and hypoketotic hypoglycemia can result in acute brain damage, apnea and in certain cases sudden fatality (8).

Few reports have assessed brain damage in 3-HMG patients using magnetic resonance imaging (MRI). Among these, predominantly cerebral white matter alternations were described $(9,10)$. The present study reports the case of a patient with a particularly severe case of $3-\mathrm{HMG}$ with prominent bilateral basal ganglia damage.

\section{Case report}

All procedures followed were in accordance with the ethical standards of the responsible committee on human experimentation of The First Hospital of Jilin University (Changchun, China) and with the Helsinki Declaration of 1975, as revised in 2000 (7). Informed consent was obtained from the parents of the patient.

A 20-month old male was admitted to The First Hospital of Jilin University with colic lasting for 3 days and onset of intermittent seizures. Although the parents reported fever of the patient, the temperature was not measured. Seizures presented as loss of consciousness, cyanosis and muscle stiffness. Five seizures were reported, and each seizure lasted 5-6 min. Prior to admission, the patient's symptoms were alleviated and consciousness was regained. The patient was the only pregnancy of his mother, and was delivered at full term without complications. At birth, the patient weighed $3.5 \mathrm{~kg}$, with a head circumference of $36 \mathrm{~cm}$ and no birth asphyxia. The child was formula-fed, and suffered from recurrent diarrhea and was frequently hospitalized, averaging once per month. The boy had a poor appetite and had iron-deficient anemia (hemoglobin, 95-105 g/l; reference, 120-160 g/l). Regarding physical growth and neurological development, the patient was 2-3 months delayed behind children of similar age. The patient was able to walk independently and call for his parents by name. There was no family history of inherited disease or hepatitis. 
Table I. Biochemical analyses of the patient at different time points of hospitalization.

\begin{tabular}{lcccr}
\hline Parameter & $\begin{array}{c}\text { Hospital } \\
\text { admission }\end{array}$ & $\begin{array}{c}\text { 2 weeks after } \\
\text { hospitalization }\end{array}$ & $\begin{array}{c}6 \text { months after } \\
\text { hospitalization }\end{array}$ & $\begin{array}{r}\text { Reference } \\
\text { range }\end{array}$ \\
\hline Blood pH & 7.34 & 7.38 & 7.41 & $7.35-7.45$ \\
$\mathrm{HCO}_{3}^{-}(\mathrm{mmol} / \mathrm{l})$ & 14 & 23 & 25 & $21-28$ \\
Base excess $(\mathrm{mmol} / \mathrm{l})$ & -11.8 & -2.5 & -2 & $-3-3$ \\
Lactic acid $(\mu \mathrm{mol} / \mathrm{l})$ & 1.5 & 0.9 & 0.3 & $0.5-1.6$ \\
Blood ammonia $(\mu \mathrm{mol} / \mathrm{l})$ & 90 & 41 & 78 & $9-47$ \\
Aspartate transaminase $(\mathrm{U} / \mathrm{l})$ & 156 & 120 & 92 & $15-40$ \\
Alanine aminotransferase $(\mathrm{U} / \mathrm{l})$ & 145 & 107 & 191 & $9-50$ \\
Glucose $(\mathrm{mmol} / \mathrm{l})$ & 3.7 & 4.8 & 4.5 & $3.9-6.1$ \\
\hline
\end{tabular}
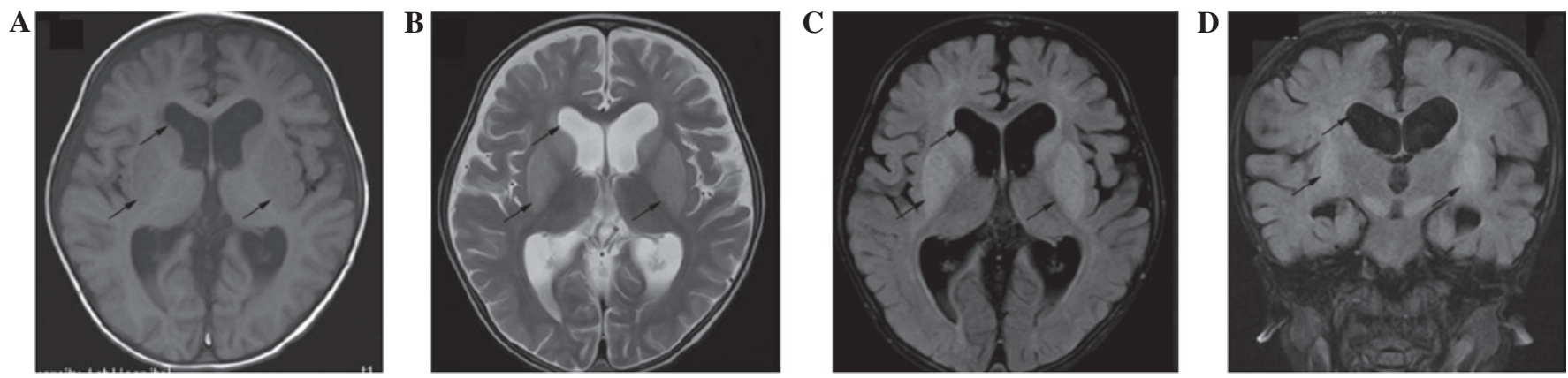

Figure 1. Magnetic resonance imaging (axial). (A) T1-weighted, (B) T2-weighted, (B) fluid-attenuated inversion recovery (FLAIR; cross section) and (D) FLAIR (coronal) images. Two lower arrows denote (A) hypointensity and (B-D) hyperintensity in the basal ganglia (bilateral), enlarged lateral ventricle (upper arrow), and widening of the temporal lobe groove bilaterally.

At the time of hospitalization, the 20 -month old was $82 \mathrm{~cm}$ tall, weighing $10 \mathrm{~kg}$ and with a head circumference of $52 \mathrm{~cm}$. On physical examination, the patient exhibited thin hair and pale skin. Patient was in a semi-coma, had slow pupillary reflex to light and slightly congested throat. The patient's muscle tension and knee reflex were weak, and the Babinski sign was negative. No abnormalities in the heart, lungs and abdomen were discerned.

During the first week of hospitalization, the patient experienced intermittent fever, averaging two or three episodes per day, with a maximum temperature of $39^{\circ} \mathrm{C}$. Seizures occurred one to two times per days with symptoms as described previously. Blood samples were collected from the child with his parents' informed consent. Routine blood tests reported: White blood cell count, $8.24 \times 10^{9} / 1$; neutrophil percentage, $0.84 \%$; lymphocyte percentage, $0.11 \%$; red blood cell count, $3.89 \times 10^{12} / 1$; hemoglobin, $86 \mathrm{~g} / 1$; hematocrit, $0.278 \mathrm{l} / 1$; mean corpuscular volume, $71.5 \mathrm{fl}$; high sensitive $\mathrm{C}$-reactive protein, $23 \mathrm{mg} / 1$ (reference, $0-3 \mathrm{mg} / \mathrm{l}$ ); procalcitonin (PCT), $54.20 \mathrm{ng} / \mathrm{ml}$ (reference, $0-0.5 \mathrm{ng} / \mathrm{ml}$ ); and urinary ketone (-), iron $7.3 \mathrm{mmol} / \mathrm{l}$ (reference, $9-22 \mathrm{mmol} / \mathrm{l})$. Ferritin, total iron binding capacity, folic acid and vitamin $\mathrm{B}_{12}$ were all within the normal range. Blood gas analysis showed metabolic acidosis, hyperammonemia, hypoglycemia and increased liver enzyme activity (Table I). HMG-CoA lyase activity in lymphocytes (Synergy H1; BioTeke Corporation, Beijing, China) was $\sim 5 \%$ of the normal activity. Ceruloplasmin level was normal, as was a routine cerebrospinal fluid biochemical test (UniCel ${ }^{\circledR}$ DxC 800 Synchron ${ }^{\circledR}$ Clinical System; Beckman Coulter, Inc., Brea, CA, USA). Results from brain axial MRI (MAGNETOM Avanto; Siemens AG, Munich, Germany) are shown in Fig. 1. White matter abnormalities were observed in the bilateral frontotemporal lobe and ventricle. In T1-weighted, T2-weighted (Fig. 1A and B), and fluid-attenuated inversion recovery images (Fig. 1C and D), marked symmetrical and bilateral patchy and focal signals in the brain stem and basal ganglia were detected (Fig. 1C and D). The ventricles were enlarged, with a widening of the groove of the anterior temporal, and parietal lobes, bilaterally (Fig. 1, upper arrow). The most prominent abnormality was a bilateral hyperintensive signal in the basal ganglia (Fig. 1B-D, lower arrows). Metabolic profiling of urine was performed using gas chromatography/mass spectrometry (GC/MS) analysis (GCMS-QP2010 Ultra; Shimadzu Corporation, Kyoto, Japan). Levels of 3-hydroxy-3-methyl-glutaric acid, 3-methyl-glutaric acid, 3-methyl-glutaconic acid, 3-hydroxy-isovaleric acid, isovaleric-glycine, 3-methyl-crotonyl-glycine, glutaric acid and hexane diacid were increased (Table II). Tandem MS analysis of blood (API 3200MD; AB SCIEX, Framingham, MA, USA) identified increased 3-hydroxy-isovaleryl-carnitine and adipoyl-carnitine levels (Table II) and normal amino acid levels. Based on the above findings, the patient was diagnosed with 3-HMG, sepsis, metabolic acidosis, and iron-deficient anemia, and was prescribed cefepime dihydrochloride monohydrate $(0.5 \mathrm{~g}$ twice daily intravenous injection; Youcare Pharmaceutical Group Co., Ltd., Beijing, China), levetiracetam 
Table II. Urine gas chromatography/mass spectrometry and blood tandem mass spectrometry analysis.

\begin{tabular}{lrl}
\hline Analyte & Value & Reference \\
\hline Urinary acid (mg/g creatinine) & & \\
3-Hydroxy-3-methyl-glutaric acid & 655.77 & $0-25.7$ \\
3-Methyl-glutaric acid & 269.67 & $0-4.5$ \\
3-Methyl-glutaconic acid & 615.28 & $0-4.2$ \\
3-Hydroxy-isovaleric acid & 413.45 & $0-2.3$ \\
Isovaleric-glycine & 1.73 & $0-0.4$ \\
3-Methyl-crotonyl-glycine & 117.34 & $0-0$ \\
Glutaric acid & 44.96 & $0-4$ \\
Hexane diacid & 71.82 & $0.5-5$ \\
Blood carnitine spectrum $(\mu$ mol/l) & & \\
3-Hydroxy-isovaleryl-carnitine & 5.04 & $0.06-0.6$ \\
Adipoyl-carnitine & 0.09 & $0-0.06$ \\
\hline
\end{tabular}

(0.125 g Keppra twice daily; UCB Pharma S.A.,Braine-l'Alleud, Belgium) and L-carnitine (0.5 ml Levocarnitine Oral Solution twice daily; Northeast Pharmaceutical Group Co., Ltd., Shenyang, China).

The patient's symptoms improved after 15 days of hospitalization. However, the patient continued to exhibit weak response to stimuli, unresponsiveness to calling and opisthotonos. Patient was maintained on a liquid diet and had no fever, vomiting, diarrhea or convulsions. Neurological examination revealed soft neck, normal muscle strength in limbs, increased muscle tension, normal knee reflex and positive Babinski sign. Blood tests showed normal levels of high sensitive C-reactive protein and PCT. Blood gas analysis showed that blood ammonia and glucose levels were normal. Liver aminotransferase activity remained elevated (Table I). The patient was discharged, and leucine-free formula and L-carnitine were prescribed. This treatment regimen, however, was not administered by the parents after discharge.

At the 6 month follow-up, the patient continued to suffer from intermittent vomiting and diarrhea. He could open and close his eyes, but was bedridden, unresponsive to calling, had unconscious limb movement and high muscle tension. The results of a blood gas analysis and the blood sugar level were normal, although liver aminotransferase activity and blood ammonia levels remained high (Table II). At the 1 year follow-up, the patient showed no significant changes and the parents did not agree to conducting review laboratory tests and MRI for economic reasons.

\section{Discussion}

The onset of 3-HMG varies between individuals, with $\sim 30 \%$ of patients displaying symptoms at the neonatal stage, while the remaining $60-70 \%$ of patients develop symptoms between 3 and 12 months after birth (11). These patients often present clinically with the following symptoms: Vomiting, diarrhea, hypotonia, hypothermia, lethargy, apnea and coma (12). The lethality rate for $3-\mathrm{HMG}$ is $\sim 20 \%$. Since early manifestations of the disease lack specificity and routine laboratory tests often fail to detect abnormality when the disease is in remission, 3-HMG is frequently misdiagnosed as septicemia or hypoglycemia (12). Diagnosis of 3-HMG requires GC/MS profiling of organic acids in urine and tandem MS analysis of blood (8). An increase in 3-hydroxy-isovaleryl-carnitine and adipoyl-carnitine in tandem MS analysis of blood and an increase in urine secretion of 3-hydroxy-3-methyl-glutaric acid, 3-methyl-glutaric acid, 3-hydroxy-isovaleric acid, and 3-methyl-glutaconic acid are indicative of 3-HMG (13). Among these, 3-hydroxy-3-methyl-glutaric acid is a specific marker for 3-HMG. The gene encoding HL is on chromosome lp36.1-p35 and consists of nine exons and eight introns. To date, $>50$ point mutations identified over the entire HL gene have been described (10). Therefore, DNA sequencing may be used to confirm a diagnosis of 3-HMG. In addition, other diagnostic methods include a skin fibrosis test and an HL activity test in lymphocytes or from a liver biopsy $(12,14)$.

The present patient had a history of recurrent diarrhea, poor feeding and was frequently hospitalized without a clear diagnosis. He arrived at The First Hospital of Jilin University with reports of infection and frequent seizures. Patient has acidosis, hepatomegaly, increase liver enzyme activity, hyperammonemia, hypoketotic hypoglycemia and low HL activity. Glutaric aciduria and Reye syndrome were eliminated as possible diagnoses; Glutaric aciduria has characteristic alterations in the GC/MS profile, including elevated concentrations of glutaryl-CoA, glutaric acid, 3-hydroxyglutaric acid and glutarylcarnitine in body tissues, whereas Reye syndrome shows normal GC/MS analyses $(15,16)$. Based on these findings, in combination with blood and urine metabolic tests, the patient was diagnosed with 3-HMG. No genetic testing was performed. Based on the levels of 3-methyl-crotonyl-glycine, glutaric acid and hexane diacid, the condition was considered to be particularly severe (5).

During an acute 3-HMG crisis, treatment includes glucose injection and rapid correction of acidosis, hyperammonemia, and liver damage (17). Administration of L-carnitine may increase the excretion of toxic acid metabolites and prevent cardiomyopathy. Since fasting and infection are triggers for 3-HMG onset, they should be avoided during remission (12). For long term maintenance, leucine-free formula and a low fat, low protein and high carbohydrate diet is recommended (18). For patients with a family history of $3-\mathrm{HMG}$, a diagnosis can be confirmed between 13 and 23 weeks of pregnancy by analyzing the organic acid content of the amniotic fluid and the urine of the mother. In addition, an HL activity test in chorionic villus cells can be performed to diagnose 3-HMG prior to birth $(5,14)$.

The mechanism underlying 3-HMG-associated brain injury is unknown. Based on MRI, the most common damage is alternation of white matter, with varying degrees of diffusion or hypointensity in the absence or presence of atrophy $(9,10)$. There was one previous report of a patient with major white matter abnormalities in the corticospinal tract on MRI (19), and another of a newborn patient with abnormal MRI signal intensity in the thalamus and basal ganglia (9). Fernandes et al (20) found that intrastriatal administration of HMG and 3-methylglutaric acid increased oxidative stress within the rat striatum, which may explain in part the cerebral alterations in HL deficient patients. In the present case, MRI revealed white matter abnormality 
and atrophy, and the most prominent lesion in basal ganglia was even more pronounced than previously reported (9). The considerable brain damage of the present patient may be due to recurrent hypoketotic hypoglycemia, damage caused by toxic leucine metabolites that passed through the under-developed blood-brain barrier. In addition, the insufficient ketone body supply in this patient may have caused myelination deficiency. Unfortunately, in this case the parents refused further treatment, which likely contributed to the further deterioration of the patient's neurological condition. At the last follow-up, six month after initial hospitalization (age, 26 months), the patient remained unresponsive to calling and his prognosis was poor.

\section{References}

1. Aoyama Y, Yamamoto T, Sakaguchi N, Ishige M, Tanaka T, Ichihara T, Ohara K, Kouzan H, Kinosada Y and Fukao T: Application of multiplex ligation-dependent probe amplification, and identification of a heterozygous Alu-associated deletion and a uniparental disomy of chromosome 1 in two patients with 3-hydroxy-3-methylglutaryl-CoA lyase deficiency. Int J Mol Med 35: 1554-1560, 2015.

2. Faull KF, Bolton PD, Halpern B, Hammond $J$ and Danks DM: The urinary organic acid profile associated with 3-hydroxy-3-methylglutaric aciduria. Clin Chim Acta 73: 553-559, 1976.

3. Ozand PT, Devol EB and Gascon GG: Neurometabolic diseases at a national referral center: Five years experience at the King Faisal specialist hospital and research centre. J Child Neurol 7 (Suppl): S4-S11, 1992.

4. Lin WD, Wang CH, Lai CC, Tsai Y, Wu JY, Chen CP and Tsai FJ: Molecular analysis of Taiwanese patients with 3-hydroxy-3-methylglutaryl CoA lyase deficiency. Clin Chim Acta 401: 33-36, 2009.

5. Ma YY, Song JQ, Wu TF, Liu YP, Xiao JX, Jiang YW and Yang YL: Leucodystrophy induced by late onset 3-hydroxy-3-methylglutaric aciduria. Zhong Guo Dang Dai Er Ke Za Zhi 13: 392-395, 2011 (In Chinese).

6. Leung AA, Chan AK, Ezekowitz JA and Leung AK: A Case of Dilated Cardiomyopathy Associated with 3-Hydroxy-3-Methylglutaryl-Coenzyme A (HMG CoA) Lyase Deficiency. Case Rep Med 2009: 183125, 2009.

7. Puisac B, Arnedo M, Casale CH, Ribate MP, Castiella T, Ramos FJ, Ribes A, Pérez-Cerdá C, Casals N, Hegardt FG and Pié J: Differential HMG-CoA lyase expression in human tissues provides clues about 3-hydroxy-3-methylglutaric aciduria. J Inherit Metab Dis 33: 405-410, 2010.

8. Hong F, Huang X, Tong F, Yang J, Yang R, Zhou X, Huang X, Mao H and Zhao Z: A family study of 3-hydroxy-3-methylglutaric aciduria with 3 cases of sudden infant death. Zhong Hua Er Ke Za Zhi 52: 397-399, 2014 (In Chinese).
9. van der Knaap MS, Bakker HD and Valk J: MR imaging and proton spectroscopy in 3-hydroxy-3-methylglutaryl coenzyme A lyase deficiency. AJNR Am J Neuroradiol 19: 378-382, 1998.

10. Funghini S, Pasquini E, Cappellini M, Donati MA, Morrone A, Fonda $\mathrm{C}$ and Zammarchi E: 3-Hydroxy-3-methylglutaric aciduria in an Italian patient is caused by a new nonsense mutation in the HMGCL gene. Mol Genet Metab 73: 268-275, 2001.

11. Puisac B, Ramos M, Arnedo M, Menao S, Gil-Rodriguez MC, Teresa-Rodrigo ME, Pié A, de Karam JC, Wesselink JJ, Giménez I, et al: Characterization of splice variants of the genes encoding human mitochondrial HMG-CoA lyase and HMG-CoA synthase, the main enzymes of the ketogenesis pathway. Mol Biol Rep 39: 4777-4785, 2012

12. Arnedo M, Ramos M, Puisac B, Gil-Rodríguez MC, Teresa E, Pié Á, et al: Advances in the study of genetic disorders. In: Mitochondrial HMG-CoA synthase deficiency. Ikehara K (ed.) InTech, pp 169-188, 2011.

13. Leipnitz G, Vargas CR and Wajner M: Disturbance of redox homeostasis as a contributing underlying pathomechanism of brain and liver alterations in 3-hydroxy-3-methylglutaryl-CoA lyase deficiency. J Inherit Metab Dis 38: 1021-1028, 2015.

14. Santarelli F, Cassanello M,Enea A, Poma F,D'Onofrio V, Guala G, Garrone G, Puccinelli P, Caruso U, Porta F and Spada M: A neonatal case of 3-hydroxy-3-methylglutaric-coenzyme A lyase deficiency. Ital J Pediatr 39: 33, 2013.

15. Boy N, Heringer J, Haege G, Glahn EM, Hoffmann GF, Garbade SF, Kölker S and Burgard P: A cross-sectional controlled developmental study of neuropsychological functions in patients with glutaricaciduria type I. Orphanet J Rare Dis 10: $163,2015$.

16. Gallucci M, Smith JD, Limbucci N, Rossi A, Demaerel P, Krings T, D'Amico A and Micheli C: Pediatric Inflammatory Diseases. Part IV: Miscellaneous, Reye, PRES, Sarcoidosis. Neuroradiol J 25: 725-738, 2012.

17. Fukao T, Mitchell G, Sass JO, Hori T, Orii K and Aoyama Y: Ketone body metabolism and its defects. J Inherit Metab Dis 37: 541-551, 2014.

18. Dos Santos Mello M, Ribas GS, Wayhs CA, Hammerschmidt T, Guerreiro GB, Favenzani JL, Sitta Â, de Moura Coelho D, Wajner $M$ and Vargas CR: Increased oxidative stress in patients with 3-hydroxy-3-methylglutaric aciduria. Mol Cell Biochem 402: 149-155, 2015

19. Yýlmaz Y, Ozdemir N, Ekinci G, Baykal T and Kocaman C: Corticospinal tract involvement in a patient with $3-\mathrm{HMG}$ coenzyme A lyase deficiency. Pediatr Neurol 35: 139-141, 2006.

20. Fernandes CG, da Rosa MS, Seminotti B, Pierozan P, Martell RW, Lagranha VL, Busanello EN and Leipnitz G: In vivo experimental evidence that the major metabolites accumulating in 3-hydroxy-3-methylglutaryl-CoA lyase deficiency induce oxidative stress in striatum of developing rats: A potential pathophysiological mechanism of striatal damage in this disorder. Mol Genet Metab 109: 144-153, 2013. 